Cita: Angelo, D.A.; Neves, A.N.; Correa, M.; Sermarine, M.; Zanetti, M.C.; Brandão, M.R.F. (2019). Propiedades Psicométricas de la Escala de Perfeccionismo en el Deporte (PPS-S) para el contexto brasileño. Cuadernos de Psicología del Deporte, Vol 19(2), 1-11

\title{
Propiedades Psicométricas de la Escala de Perfeccionismo en el Deporte (PPS-S) para el contexto brasileño
}

\author{
Propriedades Psicométricas da Escala de Perfeccionismo no Esporte \\ (PPS-S) para o contexto brasileiro
}

\section{Psychometric Properties of the Perfectionism for Sports Performance Scale (PPS-S) for the brazilian context}

\author{
Angelo, D.L. ${ }^{1}$, Neves, A.N. ${ }^{2}$, Correa, M. ${ }^{1}$, Sermarine, M. ${ }^{1}$, Zanetti, M.C. ${ }^{1}$ y Brandão, M.R.F. ${ }^{1}$ \\ ${ }^{1}$ Universidade São Judas Tadeu; ${ }^{2}$ Escola de Educação Física do Exército
}

\begin{abstract}
RESUMEN: El objetivo de este trabajo fue adaptarse interculturalmente y examinar las propiedades psicométricas de la Performance Perfectionism Scale for Sport (PPS-S) para uso en la población brasileña. Este instrumento fue basado en el modelo teórico de Hewitt y Flett y desarrollado por Hill, Appleton y Mallinson para medir el perfeccionismo en un contexto específico del desempeño deportivo. La validación del instrumento siguió las directrices para adaptaciones transculturales de instrumentos de medidas en ciencias de la salud. Después de la adaptación cultural de la escala original en inglés, el instrumento fue aplicado en 953 atletas (15-16 años). El modelo original de la escala fue probado, con análisis factorial confirmatorio, utilizando el software LISREL (versión 9.30). Los resultados mostraron un ajuste satisfactorio con 3 factores y 9 ítems $(\chi 2=116,15 \mathrm{p}<0,001$, $\mathrm{RMSEA}=0,063, \mathrm{GFI}=0,974, \mathrm{AGFI}=0,961, \mathrm{NFI}=0,937, \mathrm{CFI}=0,928, \mathrm{NNFI}=915, \chi 2 / \mathrm{gl}=4,83)$. Las pruebas satisfactorias de consistencia interna también se generaron a través del análisis de cargas factoriales y valores t. Los índices de alfa de Cronbach y confiabilidad compuesta no fueron adecuados en la variable latente que se refiere al perfeccionismo auto-orientado. La varianza media extractada carecía de buen ajuste y sólo asumió validez convergente en uno de los tres factores del instrumento. La validez discriminante también sugiere evidencia poco contundente y, por lo tanto, más estudios con otras muestras de atletas son necesarios para evaluar de forma más adecuada las propiedades psicométricas del instrumento y afirmar su uso adecuado.
\end{abstract}

Palabras clave: perfeccionismo; Brasil; psicometría; desempeño; psicología del deporte.

RESUMO: O objetivo deste trabalho foi adaptar-se Inter culturalmente e examinar as propriedades psicométricas da Escala de Perfeccionismo no Desempenho Esportivo (Performance Perfectionism Scale for Sport). Este instrumento, baseado no modelo teórico de Hewitt e Flett, foi desenvolvido por Hill, Appleton e Mallinson para medir o perfeccionismo no contexto específico do desempenho esportivo. A validação do instrumento seguiu as diretrizes para adaptações transculturais de instrumentos de medidas em ciências da saúde. Após a adaptação cultural da escala original em inglês, os dados foram coletados de 953 atletas (15-16 anos) de ambos os sexos. O 


\section{Lopes Angelo et al.}

modelo original da escala foi testado, com análise fatorial confirmatória, utilizando o software LISREL (versão 9.30). Os resultados revelaram um ajuste satisfatório com 3 fatores e 9 itens $(\chi 2=116,15 \mathrm{p}<0,001$, RMSEA $=$ $0,063, \mathrm{GFI}=0,974, \mathrm{AGFI}=0,961, \mathrm{NFI}=0,937, \mathrm{CFI}=0,928, \mathrm{NNFI}=.915, \chi 2 / \mathrm{gl}=4,83)$. Testes satisfatórios de consistência interna também foram gerados através da análise de cargas fatoriais e valores t. Contrariamente, os índices dos testes de alfa de Cronbach's e confiabilidade composta não foram adequados na variável latente que se refere ao perfeccionismo auto-orientado. A variância media extraída careceu de bom ajuste e só assumiu validade convergente em um do três fatores do instrumento. A validade discriminante também sugere evidencia pouco contundente e, portanto, mais estudos com outras amostras de atletas são necessários para avaliar de forma mais adequada as propriedades psicométricas do instrumento e afirmar seu uso adequado.

Palavras chave: perfeccionismo; Brasil; psicomentria; desempenho; psicologia do esporte.

ABSTRACT: The objective of this work was to adapt and validate psychometrically the Performance Perfectionism Scale for Sport (PPS-S) for use in the Brazilian population. This instrument was based on the theoretical model of Hewitt and Flett and developed by Hill, Appleton and Mallinson to measure perfectionism in a specific context of sports performance. The validation of the instrument followed the guidelines for transcultural adaptations of health science measurement instruments. After the intercultural adaptation of the original scale in English, the data were collected from 953 athletes (15-16 years old) of both sexes. The original scale model was tested with confirmatory factorial analysis using the LISREL software (version 9.30). The results revealed a satisfactory fit with 3 factors and 9 items $(\chi 2=116.15 \mathrm{p}<0.001$, RMSEA $=0.063$, GFI $=0.974$, AGFI $=0.961$, $\left.\mathrm{NFI}=0.937, \mathrm{CFI}=0.928, \mathrm{NNFI}=915, \chi^{2} / \mathrm{gl}=4.83\right)$. Satisfactory tests of internal consistency were also generated through the analysis of factorial loads and t-values. In contrast, Cronbach's alpha test scores and composite reliability were not adequate for the latent variable that refers to self-oriented perfectionism. The extracted mean variance lacked a good fit and only assumed convergent validity in one of the three factors of the instrument. Discriminant validity also suggests little conclusive evidence and, therefore, more studies with other samples of athletes are necessary to better evaluate the psychometric properties of the instrument and to assert its adequate use.

Keywords: perfectionism; performance; Brazil; psychometrics; sport psychology. 


\section{Propiedades Psicométricas de la PPS-S para el contexto brasileño}

\section{INTRODUCCIÓN}

Perfeccionismo es un concepto psicológico que se refiere a una tendencia del individuo de autoevaluarse con patrones excesivamente elevados y deseo de alcanzar un desempeño impecable. Es un rasgo de personalidad multidimensional, resultado de la interacción entre factores ambientales, sociales, fisiológicos, genéticos y biológicos con importantes implicaciones para los atletas (Frost, Marten, Lahart y Rosenblate, 1990; Flett y Hewitt, 2005). Los instrumentos usados para evaluar el perfeccionismo abarcan dos dimensiones de orden superior conocidas como Esfuerzos Perfeccionistas (EP) y Preocupaciones Perfeccionistas (PP). Los EP están asociados a correlatos, procesos y consecuencias deseables y adaptativas, tales como, autoconfianza, foco en la resolución de problemas y desempeño. Las PP se asocian a correlatos, procesos y consecuencias indeseables y mal adaptativas, tales como, ansiedad, protección evasiva y burnout (Madigan, 2016; González y González, 2017).

Entre los instrumentos más utilizados para evaluar perfeccionismo está la Hewitt-Multidimensional Perfectionism Scale - HMPS (Hewitt y Flett, 1991), que aborda el perfeccionismo de modo general, o sea, evalúa una gama de dominios (trabajo, educación y deporte) en tres dimensiones que incluye el perfeccionismo auto-orientado, perfeccionismo socialmente prescrito y perfeccionismo orientado hacia otros. Aunque el modelo de Hewitt y Flett (1991) presente una serie de puntos fuertes notables, la mayoría de los investigadores del deporte defienden el uso de medidas multidimensionales específicas del dominio para mejor capturar (o mensurar) el perfeccionismo (Madigan, 2016; Dunn et al., 2016).

Un instrumento desarrollado especialmente para evaluar el perfeccionismo en el contexto deportivo es la Sport-Multidimensional Perfectionism Scale-2 (SMPS-2: Gotwals et al., 2010). La SMPS-2 está basada en la Frost-Multidimensional Perfectionism Scale (FMPS: Frost, Marten, Lahart y Rosenblate, 1990), un instrumento ampliamente utilizado y validado para diferentes poblaciones (Correia, Rosado, y Serpa, 2017). La escala de Frost difiere de la de Hewitt en la forma como aborda el perfeccionismo al medir las dimensiones teóricas. $\mathrm{La}$ FMPS evalúa seis dimensiones del perfeccionismo:
Exigencias Personales (PP), Preocupación por los Errores (PE), Críticas Paternas (CP), Expectativas Paternas (EP), Dudas sobre Acciones (DA) y Organización (O), mientras que la HMPS, como mencionado anteriormente, evalúa tres dimensiones.

La SMPS-2 fue desarrollada para resolver las deficiencias encontradas en una versión anterior, la Sport-Multidimensional Perfectionism Scale (Dunn et al., 2006). En esa primera versión, dos dominios (Dudas sobre Acciones y Organización) fueron excluidos por no mostrar indicadores psicométricos adecuados. Investigaciones subsecuentes relataron la importancia de esos dos dominios para evaluar el perfeccionismo en el deporte, resultando en el desarrollo del SMPS-2, una vez que la primera versión no logró evaluar el perfeccionismo adecuadamente. Los ítems excluidos en la primera versión fueron incluidos en la SMPS-2, sin embargo, el instrumento demostró algunas inconsistencias, que vienen siendo repetidamente relatadas, con relación a la coherencia interna. Índices de ajuste no adecuados fueron encontrados al testar el modelo latente del perfeccionismo (Gotwals, Dunn, Dunn, y Gamache, 2010; Dunn et al., 2016).

Así, basado en la necesidad de un instrumento válido y confiable que capturase medidas específicas del dominio del deporte, Hill, Appleton y Mallinson (2016) desarrollaron la Performance Perfectionism Scale for Sport (PPS-S). La PPS-S se compone de 12 cuestiones que deben ser respondidas por medio de una escala Likert. Es un instrumento que mide las dimensiones del perfeccionismo basado en el modelo teórico de Hewitt y Flett (1991) y evalúa aspectos específicos del contexto deportivo con foco en el desempeño, suministrando entonces, medidas para cada una de las siguientes dimensiones: Perfeccionismo en el Desempeño Auto-Orientado (PDAO), Perfeccionismo en el Desempeño Socialmente Prescrito (PDSP) y Perfeccionismo en el Desempeño Orientado hacia Otros (PDOO). El PDAO es la tendencia de establecer patrones personales excesivamente elevados, enfocar en fallas de desempeño personal y responder a una performance deficiente con autocrítica severa, el PDSP es la percepción de que otras personas nos evalúan rigurosamente y nos presionan para que seamos perfectos y el PDOO es la tendencia a 


\section{Lopes Angelo et al.}

enfocar en las fallas de desempeño de los demás e imponer patrones perfeccionistas a los demás.

La primera etapa de validación de la PPS-S contempló tres estudios con múltiples muestras de atletas adolescentes y adultos jóvenes. Al examinar las relaciones entre las diferentes medidas de perfeccionismo (validad concurrente), correlaciones significativas fueron observadas entre la PPS-S y la SMPS-2. Correlaciones positivas fuertes fueron encontradas entre la subescala PDAO y todas las dimensiones de la SMPS-2, pero sobretodo en Patrones Personales. La PDSP y la PDOO también fueron positivamente relacionadas con todas las dimensiones del SMPS-2. Todas las correlaciones fueron bastantes consistentes $\mathrm{y}$, en la regresión múltiple, la SPMS-2 demostró ser significante predictora en todas las dimensiones de la PPS-S en las tres muestras evaluadas por los autores. Las subescalas de la PPS-S exhibieron buena confiabilidad: PDAO ( $\alpha$ de Cronbach $=.83$ a .70$)$; $\operatorname{PDSP}(\alpha=.75$ a .73$)$ y PDOO $(\alpha=.87$ a .79$)$. Las puntuaciones del test de legibilidad mostraron que la PPS-S es una herramienta apropiada para uso entre atletas adolescentes y jóvenes. Los análisis factoriales exploratorios y confirmatorio y la evaluación inicial de validez de constructo dio indicación de que la PPS-S ofrece una medida confiable y válida del perfeccionismo en el desempeño y que, debido a su brevedad, puede ser fácilmente utilizada (Hill, Appleton y Mallinson, 2016).

Nascimento, Nickeng, Lavallee y Fiorese (2015) hicieron la adaptación y validación transcultural de la SMPS-2 para uso en el contexto deportivo brasileño, sin embargo, pese el rigor con que la escala fue validada, se verificaron inconsistencias en los modelos del análisis factorial confirmatorio y el instrumento perdió cerca de $57 \%$ de los ítems originales. A pesar de haber perdido la mitad de los elementos originales, la escala SMPS-2 es un instrumento extenso y en ambientes deportivos, escalas más cortas pueden ser ventajosas, pues los atletas generalmente tienen poca disponibilidad para responderlas. Aunque abordan una perspectiva más restringida del constructo, escalas más cortas, pueden minimizar el tiempo de respuesta, el cansancio de los participantes y la pérdida de información por cuenta de respuestas que faltan (Liu y Tenenbaum, 2018; Weinberg y Gould, 2018). Estos motivos lo que nos llevó a testar las evidencias de validad de la PPS-S para el contexto deportivo brasileño. Por lo tanto, nuestra intención fue analizar las propiedades psicométricas de la Performance Perfectionism Scale for Sport en su versión en portugués para el contexto deportivo brasileño, con el fin de ofrecer un instrumento psicométricamente válido y confiable y que puede ser fácilmente utilizada por su brevedad para poblaciones de atletas adolescentes y jóvenes.

Las propiedades psicométricas de la PPS-S adaptada para el contexto brasileño fueron testadas, respetando los procedimientos adoptados por los autores en el instrumento original. El estudio ofrece la adaptación cultural y prevé un análisis más profundo de la estructura del instrumento utilizando análisis factorial confirmatorio, evidencia de validez de constructo y confiabilidad interna para una muestra de atletas adolescentes.

\section{MÉTODO}

\section{Participantes}

Participaron del estudio 953 atletas adolecentes (483 mujeres, 470 hombres) con edades entre 15 y 16 años (Media=15.8; $\mathrm{DP}=.8$ ) y que practicaban las siguientes modalidades: Fútbol $(\mathrm{n}=190)$, futsal $(\mathrm{n}=$ 37), baloncesto $(n=121)$, atletismo $(n=27)$, voleibol $(\mathrm{n}=98)$, balonmano $(\mathrm{n}=158)$, rugby $(\mathrm{n}=54)$, hockey $(\mathrm{n}=17)$, judo $(\mathrm{n}=54)$, karate $(\mathrm{n}=20)$, taekwondo $(n=18)$ y boxeo $(n=11)$, ciclismo $(n=7)$, tenis $(n=37)$, natación $(\mathrm{n}=54)$, gimnasia artística $(\mathrm{n}=3)$, gimnasia rítmica $(\mathrm{n}=38)$ y $\mathrm{BMX}(\mathrm{n}=9)$. Los atletas entrenaban un promedio de 2.8 horas por día $(\mathrm{DP}=1.1), 4$ días por semana $(\mathrm{DP}=1.0)$ y tenían 6 años $(\mathrm{DP}=3.1)$ de experiencia en la modalidad. Los datos fueron recolectados en clubes y centros de entrenamiento ubicados en las ciudades de São Paulo y São José do Campos.

\section{Adaptación cultural de la PPS-S}

Fue solicitada autorización formal para la traducción y validación de la Performance Perfectionism ScaleSport (PPS-S) para la población brasileña al Profesor Dr. Andrew Hill, autor del instrumento original en el Reino Unido. La traducción respetó las directrices para adaptaciones transculturales de instrumentos de medidas en ciencias de la salud (Beaton, Bombardier, Guillemin y Ferraz, 2000) y está conformada por siete pasos: (1) traducción del instrumento del Inglés 


\section{Propiedades Psicométricas de la PPS-S para el contexto brasileño}

para el Portugués por dos profesionales de los dos países y que dominan la Lengua Inglesa, generando así dos versiones (T1 y T2) del instrumento; (2) síntesis de las dos versiones para la elaboración de una nueva versión T3 del instrumento; (3) traducción inversa del T3 para el Inglés por dos individuos que tienen el Inglés como lengua nativa y que dominan la Lengua Portuguesa, generando las versiones T4 y T5 del instrumento; (4) síntesis de las dos versiones para la elaboración de una nueva versión T6 del instrumento; (5) evaluación de la versión T6 del instrumento por doctores especialistas, generando una nueva versión T7; (6) envió de la versión T7 del instrumento para el autor del instrumento original para que ele pudiese dar su opinión como experto; (7) elaboración de la versión final T8 del instrumento.

La versión pre-test fue presentada a 6 participantes (3 mujeres, 3 hombres) con edades entre 15 y 16 años reclutados entre atletas del club. Cada participante completó la escala de pre-prueba y luego participó en una entrevista para verificar la comprensión de los elementos e instrucciones, la adecuación de los diseños y la congruencia entre la respuesta deseada y la respuesta indicada. El pre-test no indicó necesidad de revisión. Por medio de este proceso, la versión del instrumento para el contexto brasileño estaba lista para la recolección de datos.

\section{Procedimiento}

El presente estudio recibió autorización del autor del PPS-S y aprobación del Comité de Ética en Investigación de la Universidad São Judas Tadeu (69496017.1.0000.0089/2017). El reclutamiento de los participantes no fue probabilístico y los investigadores fueron a los clubes y centros de entrenamiento ubicados en las ciudades de São Paulo y São José do Campos, con el fin de reclutar a los participantes. Después de recibir información sobre los procedimientos y objetivos de la investigación, los atletas fueron invitados a participar. Todos los participantes, incluidos los menores de edad, firmaron los formularios de consentimiento. Los padres de los participantes menores de 18 años fueron informados sobre la naturaleza del estudio $\mathrm{y}$, después de concordar con la participación de sus hijos, firmaron los formularios de consentimiento. Se marcó el día y la hora para la aplicación de los cuestionarios. En el día programado, aquellos que quisieron participar del estudio recibieron una explicación sobre los procedimientos que se adoptarán y la importancia de responder a los instrumentos de la manera más honesta y sincera posible, pero caso el participante no se sintiera a gusto, podría interrumpir su participación en la investigación en cualquier momento, sin ningún perjuicio. Finalmente, la aplicación de los instrumentos se realizó en el lugar de formación de los atletas, en un entorno adecuado y cómodo, siempre con la orientación de un investigador.

\section{Análisis estadístico}

El modelo fue testado, usándose análisis confirmatorios, por medio del software LISREL en la versión PRELIS TM2. Fue adoptado el criterio de exclusión listwise para eliminación de casos con respuestas faltantes (Joreskog y Sorbom, 2006). Tras generar el archivo PRELIS TM2, fue utilizado el modelo SIMPLIS, que es una codificación del sistema LISREL ${ }^{\circledR}$ El Análisis Factorial Confirmatorio fue entonces realizado, posibilitando evaluar los parámetros del constructo del modelo de mensuración. Para estimar los modelos, fue utilizado el método del Mínimo Cuadrado No Ponderado (Holgado-tello, 2015). Consideramos los siguientes índices para el ajuste de modelos: índice de calidad del ajuste (GFI), índice ajustado de calidad del ajuste (AGFI) e índice de ajuste comparativo (CFI). De acuerdo con la literatura (Hair, Babin, Anderson y Black, 2018) estos índices deben ser iguales o superiores a .09. Fue también considerada la raíz del error cuadrático medio de aproximación (RMSEA), cuyo valor establecido de aceptación debe estar abajo de .08 .

E1 Análisis Factorial Confirmatorio (AFC) es típicamente adoptado en esta fase del proceso de validación, es un análisis popular porque permite a los investigadores testar la estructura de los factores entre los ítems y las dimensiones de la escala. Además, permite una vigorosa prueba de estructura de factor en términos de ajuste siendo, por lo tanto, un análisis robusto para validar instrumentos psicométricos.

Para evaluar el modelo de medición, se examinó la validez del constructo (discriminante y convergente). Para establecer la validez convergente, se analizaron 


\section{Lopes Angelo et al.}

los valores $t$, las cargas factoriales de las variables observables y la Varianza Media Extractada (VME). Cargas factoriales mayores que .50 y valores- $t$ mayores o igual a 1.96 fueron considerados aceptables (Hair, Babin, Anderson y Black, 2018). Para VME, valor mayor que .50 indica validez convergente adecuada de los indicadores individuales y del constructo (Ramos et al., 2017).

Para la validad discriminante se hizo la comparación entre la VME y la correlación al cuadrado de cada factor. Los valores superiores de la primera indican que el modelo presenta buena validez discriminante (Farrell, 2010).

La consistencia interna fue determinada por la prueba alfa de Cronbach (Avecillas y Lozano, 2016). De modo general, se asume que el valor del alfa de Cronbach deba ser igual o mayor que .70, sin embargo, valores menores pueden ser igualmente relevantes de acuerdo con la naturaleza del constructo estudiado. Si la prueba tiene fines de diagnóstico, se hace necesario que el alfa alcance el índice de .70, pudiendo variar hasta .60 , en el caso de investigación (Wang y Gorenstein, 2013). Además, se calculó la fiabilidad compuesta del constructo, como un indicador de consistencia interna por la fórmula: $\mathrm{fc}=(\Sigma \lambda \mathrm{i})^{2} /(\Sigma \lambda \mathrm{i})^{2}+\Sigma \varepsilon \mathrm{i}$. Los valores mayores o iguales a .70 son preferibles (Hair, Babin, Anderson y Black, 2018) pero hasta .60, son aceptables (Bagozzi y Yi, 1988).

Por último, para saber si la configuración y los parámetros de la PPS-S son invariantes para diferentes grupos, utilizamos una técnica del modelado de ecuaciones estructurales, el Análisis
Factorial Confirmatorio Multigrupo con el objetivo de validar el instrumento por sexo. Este análisis es importante pues, las diferencias entre los grupos pueden estar relacionadas no con las diferencias reales del trazado latente, pero con parámetros no equivalentes del instrumento (Bandeira-de-mello, 2014).

\section{RESULTADOS}

\section{Análisis Factorial Confirmatorio}

Los resultados iniciales mostraron ajustes pobres $(\chi 2$ $=232.45, \mathrm{p}<.001 ; \mathrm{RMSEA}=.93, \mathrm{GFI}=.982$, AGFI $=.972, \mathrm{NFI}=.859, \mathrm{CFI}=.877, \mathrm{NNFI}=.840, \chi 2 / \mathrm{gl}$ $=5.65)$. En el intento de adecuar este modelo inicial, basado en el cuestionario original, se retiró individualmente del modelo los ítems que presentaban menor carga factorial en el constructo. Se tomaron los apartados ítems $2(\lambda=0,40), 4(\lambda=$ $0,37)$ y $6(\lambda=0,38)$. Al final de este proceso, los resultados encontrados revelaron un ajuste satisfactorio con 3 factores y 9 ítems $(\chi 2=116.15 \mathrm{p}$ $<.001$; RMSEA $=.063$; GFI $=.974$, AGFI $=.961$, $\mathrm{NFI}=.937, \mathrm{CFI}=.928, \mathrm{NNFI}=.915, \chi 2 / \mathrm{gl}=4.83$ ). El valor del RMSEA indicó ajustamiento aceptable de la estructura, bien como el CFI, NFI y NNFI. Cuando verificados los índices de modificación, los análisis de post hoc no sugirieron re especificaciones sustanciales, a punto de mejorar el ajuste del modelo en ese nivel. Así, la estructura de 3 factores se mantuvo. Este modelo nacional se mostró un modelo rival más adherente que el modelo original, habiendo sido escogido como el modelo de la versión brasileña del PPS-S (Figura 1). 


\section{Propiedades Psicométricas de la PPS-S para el contexto brasileño}

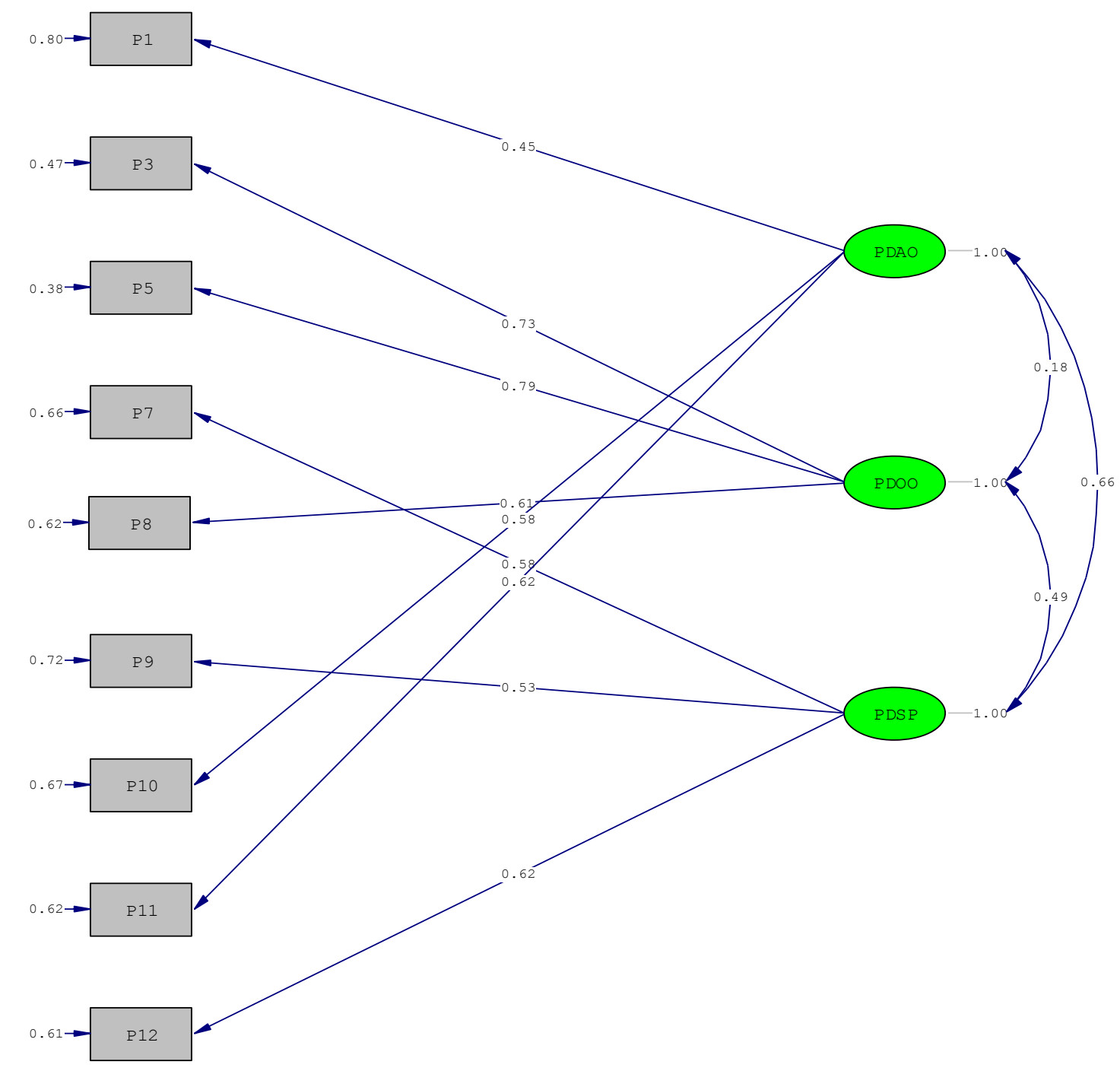

Chi-Square $=116.15, \mathrm{df}=24, \mathrm{P}-\mathrm{value}=0.00000, \mathrm{RMSEA}=0.063$

Figura 1. Modelo estructural de la versión brasileña de PPS-S

Consistencia interna, validez discriminante $y$ convergente

En cuanto a la validez, todas las cargas factoriales de la versión brasileña fueron por encima de .40 , siendo $\lambda=.45$ (ítem 1) la menor y $\lambda=.79$ la mayor carga (ítem 5). Todos los valores $t$ tuvieron significancia estadística por encima de 1.96 (Tabla 1). La VME, utilizada como indicador de validez convergente, mostró valor aceptable sólo en la variable latente PDOO, lo que indica que el constructo explica más de la mitad de la varianza de todos os indicadores que lo componen. En la comparación entre la VME y la correlación al cuadrado de cada factor, sólo la variable latente PDOO fue superior al cuadrado de la correlación entre ellas, lo que indica que el modelo no presenta buena validez discriminante. En cuanto a las medidas de confiabilidad interna, el alfa de Cronbach y la Fiabilidad Compuesta de los constructos presentaron resultados satisfactorios sólo en las variables latentes PDOO y PDSP (Tabla 2) 


\section{Lopes Angelo et al.}

Tabla 1

Medias, Desviación Estándar, cargas factoriales y t-valor de los ítems de la PPS-S versión brasileña

\begin{tabular}{|c|c|c|c|c|}
\hline Ítems PPS-S & Media & $\begin{array}{c}\text { Desviación } \\
\text { Estándar }\end{array}$ & $\begin{array}{c}\text { Cargas } \\
\text { Factoriales }\end{array}$ & t-valor \\
\hline 1.Soy muy duro conmigo cuando mi desempeño no es perfecto. & 5.41 & 1.70 & .45 & 114.22 \\
\hline $\begin{array}{l}\text { 3.Tengo una mala opinión sobre los demás cuando no se desempeñan } \\
\text { perfectamente. }\end{array}$ & 2.69 & 1.83 & .73 & 132.03 \\
\hline $\begin{array}{l}\text { 5.Tengo pensamientos negativos acerca de las personas que no se } \\
\text { desempeñan perfectamente. }\end{array}$ & 2.42 & 1.73 & .79 & 131.95 \\
\hline 7.La gente siempre espera que mi desempeño sea perfecto. & 4.51 & 1.95 & .58 & 151.35 \\
\hline 8. Yo critico a las personas si no se desempeñan perfectamente. & 2.11 & 1.54 & .61 & 115.53 \\
\hline 9.Las personas ven negativamente incluso mis mejores desempeños. & 3.10 & 1.92 & .53 & 133.48 \\
\hline 10. Yo sólo pienso bien de mí cuando mi desempeño es perfecto. & 5.91 & 1.41 & .58 & 177.07 \\
\hline 11.Para alcanzar mis metas necesito un desempeño perfecto. & 5.78 & 1.61 & .62 & 182.69 \\
\hline 12.La gente me criticó si no tengo un desempeño perfecto. & 3.61 & 2.04 & .62 & 146.66 \\
\hline
\end{tabular}

Tabla 2

Consistencia interna, validez discriminante y convergente de la PPS-S versión brasileña

\begin{tabular}{lccccc}
\hline & PDOO & PDAO & PDSP & $\alpha$ & fc \\
\hline PDOO & $.51^{*}$ & & & .80 & .75 \\
PDAO & $.03^{* *}$ & $.31^{*}$ & & .65 & .57 \\
PDSP & $.24^{* *}$ & $.43^{* *}$ & $.33^{*}$ & .70 & .60 \\
\hline
\end{tabular}

Nota. $*$ VME; $* * \mathrm{R}^{2} ; \alpha=$ alfa de Cronbach; fc $=$ Fiabilidad Compuesta

\section{Análisis de Invariancia}

Adicionalmente, probamos la invariancia del modelo entre los sexos a través del análisis multigrupos (Marôco, 2014). La invariancia configuracional del modelo modificado de la PPS-S presentó un ajuste adecuado entre atletas del sexo masculino y del sexo femenino $[\chi 2(50)=144.09 ; \chi 2 / \mathrm{gl}=2.88 ; \mathrm{CFI}$ $=.965 ; \mathrm{GFI}=.997 ; \mathrm{TLI}=.950 ;$ RMSEA $=.063$; $\mathrm{p}(\mathrm{rmsea}<.05)=.037]$, indicando que la estructura propuesta se mantiene estable en los dos grupos. El chi-cuadrado de Satorra-Bentler fue utilizado para corregir una inflación de la estadística del chicuadrado que ocurre cuando los datos son no normales multivariados (Saris y Satorra, 2018). Los índices de ajuste del modelo restringido no diferían significativamente del modelo con parámetros libres [ $\chi 2$ diff (20) 33,88, p = .090], demostrando la equivalencia de medida entre los sexos. Sin embargo, la invariancia estructural del modelo no se obtuvo $\left[\chi^{2}\right.$ $(33)=53.63, \mathrm{p}=.012]$ indicando que los niveles de correlación entre los factores no son los mismos para hombres y mujeres. Tales resultados demuestran la invariancia parcial de la versión brasileña de la PPS$\mathrm{S}$, pues sólo la invariancia configuracional y métrica fueron confirmadas.

\section{DISCUSIÓN}

En este manuscrito fueron relatados los procedimientos utilizados para adaptar y validar la Performance Perfectionism Scale for Sport para uso en la población brasileña. Comprender las dimensiones del perfeccionismo y su potencial en influenciar positivamente $o$ negativamente el comportamiento, se convierte en un importante esfuerzo de investigación, especialmente en ambientes competitivos que exigen la perfección por parte del atleta. Los instrumentos específicos en contextos específicos pueden proporcionar una mayor comprensión del fenómeno. Las investigaciones con poblaciones no deportivas mostraron la naturaleza 


\section{Propiedades Psicométricas de la PPS-S para el contexto brasileño}

predominantemente mal adaptativa del POO y del PSP asociadas a dificultades psicosociales, como el estrés relacionado al trabajo (Lizmore, Dunn y Dunn, 2017). En el contexto deportivo no se sabe si lo mismo ocurre, de ahí la importancia de examinar la naturaleza y la función del perfeccionismo en el deporte competitivo (Pineda, Morquecho y Gadea, 2018).

En los datos analizados, se constató que, de los 3 ítems inicialmente incluidos en el modelo de AFC, 2 presentaron problemas. El mismo tipo de resultado se verificó en la versión original de la escala, pero la exclusión de estos elementos no interfirió en los indicadores de adecuación del modelo. En el caso de la versión brasileña, los ítems influenciaban la estructura factorial, razón por la cual fueron excluidos.

El análisis mostró buena confiabilidad interna en la muestra analizada. Las cargas factoriales y los valores $t$ han sido satisfactorios. Los resultados del alfa de Cronbach e Fiabilidad Composta fueron abajo de lo esperado, sin embargo, se sabe que todos los indicadores de precisión tienden a aumentar con el aumento de ítems en el modelo. La cantidad de ítems de un instrumento puede afectar el valor del alfa de Cronbach y Fiabilidad Composta, luego, cuanto mayor es el número de ítems, mayor será el coeficiente. De este modo, es posible construir una escala con mayor valor de confiabilidad simplemente aumentando el número de ítems (Wang y Gorenstein, 2013). Por lo tanto, los investigadores deben estar atentos a la relatividad de los puntos de corte, pues es posible que estén validando un modelo incluso con cargas factoriales bajas si tienen un elevado número de ítems (Valentini y Damásio, 2016). Luego, aunque son inferiores a .70, los valores de alfa de la PDAO fueron igualmente relevantes por tratar-se de una escala con pocos ítems (apenas 3 ítems en cada factor), lo que puede haber llevado a valores alfa de Cronbach y Fiabilidad Composta abajo del esperado. Nuestros análisis corroboraron con los hallazgos de los autores de la PPS-S, sugiriendo que los ítems de la PDAO pueden necesitar de pequeñas revisiones para mejorar la legibilidad a medida que la validación del PPS-S continua.

La validez convergente, estimada por la varianza media extraída de las variables latentes no fue adecuada a la norma recomendada. La validez discriminante fue satisfactoria sólo en una de las tres variables latentes, siendo necesarios más estudios para evaluar adecuadamente las propiedades psicométricas del instrumento y poder afirmar su uso en atletas brasileños.

Hubo adecuación del modelo de la PPS-S evidenciada con el análisis de invariancia por sexo. Los resultados indicaron un ajuste estable sin diferencias entre los sexos. Sin embargo, los niveles de correlación entre los factores no son los mismos para hombres y mujeres indicando que la invariancia estructural no fue obtenida (Byrne, 2010). Pero aun así, la invariancia estructural no evalúa la variabilidad de las propiedades psicométricas del instrumento, no comprometiendo así la estructura factorial y la confiabilidad del modelo (Sass, 2011). Con relación a la estructura factorial, todos los 9 ítems permanecieron en la versión brasileña de la PPS-S y la estructura de 3 factores fue confirmada con índices de ajuste satisfactorios proporcionando una escala corta y fiable para que los entrenadores e investigadores puedan rastrear las dimensiones del perfeccionismo en el contexto brasileño, sin embargo, las propiedades psicométricas son cuestionables, pues, a pesar de poseer estructura factorial adecuada, la validez discriminante $\mathrm{y}$ divergente y la fiabilidad compuesta no satisface las relaciones asumidas. Así, los resultados permiten el uso cauteloso del instrumento.

\section{APLICACIONES PRÁCTICAS}

Reexaminar las propiedades psicométricas de los instrumentos adaptados validados es relevante tanto para la investigación y para el trabajo de campo. En términos prácticos, el desempeño es una de las características que definen el dominio del deporte y tal vez sea el aspecto más importante de la vida de un atleta. El uso de la escala podría ayudar a comprender cuales las dimensiones del perfeccionismo que hacen con que el atleta sea vulnerable a las dificultades y ofrece pistas para estrategias que deberían ser abordadas en el entrenamiento psicológico. Además, tales informaciones permitirán más investigaciones sobre el perfeccionismo deportivo no apenas en Brasil, pero también la posibilidad de conducir investigaciones culturales. Así, considerase que la PPS-S ofrece un medio de evaluar el perfeccionismo de desempeño en la población brasileña, que pasará a 


\section{Lopes Angelo et al.}

ser denominada Escala de Perfeccionismo no Esporte para o Contexto Brasileiro (PPS-S-BR).

\section{REFERENCIAS}

1. Avecillas, D. \& Lozano, C. (2016). Medición de la Confiabilidad del Aprendizaje del Programa R Studio Mediante Alfa de Cronbach. Revista Politécnica, 37(2), 1-9.

2. Bagozzi, R. \& Yi, Y. (1988). On thee valuation of structural equation models. Jams, 16(1), 7494.

https://doi.org/https://doi.org/10.1007/BF027233 $\underline{27}$

3. Bandeira-de-mello, R. (2014). Recursos para inovação e desempenho: uma análise da invariância de mensuração em firmas de setores de alta intensidade tecnológica no Brasil. Revista de Administração e Inovação, 11(4), 33-57. https://doi.org/10.11606/rai.v11i4.100272

4. Beaton, D. E., Bombardier, C., Guillemin, F. \& Ferraz, M. B. (2000). Guidelines for the Process of Cross-Cultural Adaptation of Self-Report Measures. Spine, 25(24), 3186-3191. https://doi.org/10.1097/00007632-20001215000014

5. Correia, M., Rosado, A., \& Serpa, S. (2017). Psychometric properties of the Portuguese version of the Frost Multidimensional Perfectionism Scale. International Journal of Psychological Research, 10(1), 8-17. https://doi.org/10.21500/20112084.2109

6. Dunn, J. G. H., Causgrove, E., Gotwals, J. K., Vallance, J. K. H., Craft, J. M., \& Syrotuik, D. G. (2006). Establishing construct validity evidence for the Sport Multidimensional Perfectionism Scale. Psychology of Sport and Exercise, 7(1), 57-79.

https://doi.org/10.1016/j.psychsport.2005.04.003

7. Dunn, J. G. H., Gotwals, J. K., Dunn, J. C., Selzler, A. M., Lizmore, M. R., Vaartstra, M. \& Gamache, V. E. (2016). A multi-sample investigation of the higher-order latent dimensionality of the Sport-Multidimensional Perfectionism Scale-2. Psychology of Sport and
Exercise, $\quad 27(8), \quad 150-156$. https://doi.org/10.1016/j.psychsport.2016.08.006

8. Farrell, A. M. (2010). Insufficient discriminant validity: A comment on Bove, Pervan, Beatty and Shiu (2009). Journal of Business Research, 63(3), 324-327.

https://doi.org/10.1016/j.jbusres.2009.05.003

9. Flett, G. L., \& Hewitt, P. L. (2005). The perils of perfectionism in sports and exercise. Current Directions in Psychological Science, 14(1), 14$18 . \quad$ https://doi.org/10.1111/j.0963$\underline{7214.2005 .00326 . x}$

10. Frost, R. O., Marten, P., Lahart, C. \& Rosenblate, R. (1990). Thedimensions of perfectionism. Cognitive Therapy and Research, 14(5), 449468. https://doi.org/10.1007/BF01172967

11. González, J. y González, A. (2017). Perfeccionismo y "alarma adaptativa" a la ansiedad en deportes de combate. Revista de Psicología del Deporte, 26(Supl. 2), 15-23.

12. Gotwals, J. K., Dunn, J. G. H., Dunn, J. C., \& Gamache, V. (2010). Establishing validity evidence for the Sport Multidimensional Perfectionism Scale-2 in intercollegiate sport. Psychology of Sport and Exercise, 11(6), 423432.

https://doi.org/10.1016/j.psychsport.2010.04.013

13. Hair, J., Babin, B. Anderson, R., \& Black, W. (2018). Multivariate data analysis (8nd ed.). Hampshire: Cengage Learning.

14. Hewitt, P. L., \& Flett, G. L. (1991). Perfectionism in the self and social contexts: Conceptualization, assessment and association with psychopathology. Journal of Personality and Social Psychology, 60(3), 456-470. https://doi.org/10.1037/t04592-000

15. Hill, A. P., Appleton, P. R., \& Mallinson, S. H. (2016). Development and Initial Validation of the Performance Perfectionism Scale for Sport (PPSS). Journal of Psychoeducational Assessment, 6(8), 42-48. https://doi.org/10.13580/j.cnki.fstc.2016.03.008 


\section{Propiedades Psicométricas de la PPS-S para el contexto brasileño}

16. Holgado-tello, F. (2015). Confirmatory factor analysis : recommendations for unweighted least squares method related to Chi-Square and RMSEA Type I error. Accion Psicologica, 12(6), 79-90.

17. Joreskog, K. G. \& Sorbom, D. (2006). Lisrel 8.8: Interactive Lisrel: Technical support. Mooresville, IN: Scientific Software.

18. Liu, S. \& Tenenbaum, G. (2018). Research Methods in Sport and Exercise Psychology. Oxford: Oxford University Press. https://doi.org/10.1093/acrefore/9780190236557. $\underline{013.224}$

19. Madigan, D. J. (2016). Confirmatory factor analysis of the Multidimensional Inventory of Perfectionism in Sport. Psychology of Sport and Exercise, 26(6), 48-51. https://doi.org/10.1016/j.psychsport.2016.06.003

20. Marôco, J. (2014). Análise de Equações Estruturais: Fundamentos teóricos, Software e Aplicações (2a ed.). São Paulo: ReportNumber.

21. Nascimento, J. R. A. do, Nickeng V. J. R., Lavallee, D. \& Fiorese V. L. (2015). Adaptation and validation of the Sport Multidimensional Perfectionism Scale-2 (SMPS-2) for the Brazilian sport context. Motriz: Revista de Educação Física, 21(2), 125-136. https://doi.org/10.1590/S1980$\underline{65742015000200003}$

22. Pineda, H. A., Morquecho, R. \& Gadea, E. (2018). Evidencias de validez test-criterio en el uso de la Escala Multidimensional de Perfeccionismo en el Deporte-2. Cuadernos de Psicología Del Deporte, 18(3), 129-140.

23. Lizmore, M., Dunn, J. G. H., \& Dunn, J. C. (2017). Perfectionistic strivings, perfectionistic concerns, and reactions to poor personal performances among inter collegiate athletes. Psychology of Sport and Exercise, 33(11), 75-84. https://doi.org/10.1016/j.psychsport.2017.07.010

24. Ramos, A., Rosado, A., Serpa, S., Cangas, A., Gallego, J., \& Ramos, L. (2017). Validity evidence of the Portuguese version of the five facet mindfulness questionnaire. Revista de Psicologia Del Deporte, 27(2), 87-98. https://doi.org/10.1590/S0102$\underline{37722014000300009}$

25. Saris, W. \& Satorra, A. (2018). The Pooled Data Approach for the Estimation of Split-Ballot Multitrait. Structural Equation Modeling: A Multidisciplinary Journal, 2, 1-14. https://doi.org/10.1080/10705511.2018.1431543

26. Sass, D. A. (2011). Testing measurement invariance and comparing latent factor means within a confirmatory factor analysis framework. Journal of Psychoeducational Assessment, 29(4), 347-363. https://doi.org/10.1177/0734282911406661

27. Valentini, F. \& Damásio, B. F. (2016). Variância Média Extraída e Confiabilidade Composta: Indicadores de Precisão. In Psicologia: Teoria e Pesquisa, 32, 1-7. https://doi.org/10.1590/0102$\underline{3772 \mathrm{e} 322225}$

28. Wang, Y. P., \& Gorenstein, C. (2013). Psychometric properties of the Beck Depression Inventory-II: a comprehensive review. Revista Brasileira de Psiquiatria, 35(4), 416-431. https://doi.org/10.1590/1516-4446-2012-1048

29. Weinberg, R. S. \& Gould, D. S. (2018). Foundations of sport and exercise psychology. (7nd ed.). Illinois: Human Kinetics. 